\title{
Ultrastructural study of the neovagina following the utilization of human amniotic membrane for treatment of congenital absence of the vagina
}

L.F. Bleggi-Torres ${ }^{1}$,

B. Werner ${ }^{1}$ and M.J. Piazza²

\author{
'Seção de Neuropatologia e Microscopia Eletrônica, Serviço de Anatomia Patológica \\ and ${ }^{2}$ Departamento de Ginecologia e Obstetrícia, Hospital de Clínicas, \\ Universidade Federal do Paraná, 80060-900 Curitiba, PR, Brasil
}

\section{Correspondence \\ L.F. Bleggi-Torres \\ Seção de Neuropatologia e \\ Microscopia Eletrônica \\ Serviço de Anatomia Patológica \\ Hospital de Clínicas, UFPR \\ Rua General Carneiro, 181 \\ 80060-900 Curitiba, PR \\ Brasil \\ Fax: 55 (041) 264-2513}

Received September 26, 1996 Accepted May 19, 1997

\begin{abstract}
We present an ultrastructural study of the utilization of human amniotic membrane in the treatment of congenital absence of the vagina in 10 patients. All patients were surgically treated with application of an amniotic membrane graft using the modified McIndoe and Bannister technique. Sixty days after surgery, samples of the vaginal neoepithelium were collected for transmission electron microscopy analysis. The ultrastructural findings consisted of a lining of mature squamous epithelium indicating the occurrence of metaplasia of the amniotic epithelium into the vaginal epithelium. The cells were arranged in layers as in the normal vaginal epithelium, i.e., superficial, intermediate and deep layers. There were desmosomes and cytoplasmic intermediate cytokeratin filaments, as well as some remnant features of the previous amniotic epithelium. These findings suggest that human amniotic membrane is able to complete metaplasia into squamous cells but the mechanism of this cellular transformation is unknown.
\end{abstract}

Key words

- Amniotic membrane

- Amnion

- Electron microscopy

- Congenital absence

of vagina

- Neovagina

\section{Introduction}

Vagina malformations are unusual but give rise to serious problems for the sexual life of the patients. Their severity ranges from vaginal shortening in patients with androgen insensitivity to complete agenesis of the vagina. Masculinized females have more complicated abnormalities and vaginal strictures, often secondary to vaginal surgery, which can be a source of major concern. Intercourse becomes difficult or impossible and when a functioning uterus is present, hematometra or hematocolpos may transform the situation into a surgical emergency.

Various treatments have been described for vaginal agenesis. Gradual vaginal dilatation, vulvovaginoplasty with labia and splitskin graft to line a surgically dissected vagina are some of the treatments proposed (1). In addition to its use as a biological dressing for wounds and burns, human amnion has also been used as a graft for surgical treatment of congenital absence of the vagina (17).

We present an ultrastructural study of the 
tissue formed after the application of amnion onto the surface of the artificially produced vaginal canal in 10 patients with congenital absence of the vagina. To our knowledge, this is the first electron microscopy study that analyzes the morphological aspect of the new epithelium lining the vagina.

\section{Patients and Methods}

Ten patients with congenital absence of the vagina seen at the Departamento de Ginecologia e Obstetrícia of Hospital de Clínicas, Curitiba, Brazil, were selected for this study. All patients were treated surgically with an amniotic membrane graft using the modified McIndoe and Bannister technique (8). Sixty days after surgery, samples of the vaginal neo-epithelium were obtained for transmission electron microscopy examination.

The amnion to be used for the graft was removed from the placenta at the time of delivery, either vaginal or by cesarean section, from 10 patients previously selected as amniotic membrane donors. Fetal membranes with meconium staining, suspected chorioamnionitis or premature rupture were not used for grafting. Before the study, sera from all amnion donors were tested for syphilis, toxoplasmosis, hepatitis $\mathrm{B}$, hepatitis $\mathrm{C}$ and HIV. Maternal blood and other contaminants were removed by washing the membrane in sterile saline until apparently clean. The membranes were stored at $4^{\circ} \mathrm{C}$ in a solution containing $80 \mathrm{mg}$ gentamicin per $100 \mathrm{ml}$ sterile saline for 48 to $72 \mathrm{~h}$ before its utilization in surgery. The membrane was applied to a vaginal latex mold in such a way that the mesenchymal surface of the amnion contacted the host tissue. Amniotic membranes from 10 patients were also submitted to transmission electron microscopy analysis for comparison.

Under general anesthesia, the patient was placed in the lithotomy position. An oblique incision was made in the vesicorectal space, with care taken to avoid injury to the bladder and rectum. The vaginal tunnel was then created by blunt dissection to a depth of 14 to $16 \mathrm{~cm}$ and a diameter of 3 to $4 \mathrm{~cm}$. After establishing homeostasis, the mold covered with a condom wrapped in amniotic membrane was inserted. Two to three silk sutures in the labia majora maintained the mold in position. The labial stitches were removed on the 8th day and the mold was withdrawn. The patient was instructed how to use a wooden vaginal dilator wrapped with a lubricated condom of appropriate size and was asked to insert it two to three times a day for at least $10 \mathrm{~min}$. Three weeks later, the patient was encouraged to have intercourse, if appropriate, or to continue with the dilator one week longer.

Sixty days after surgery, samples were taken from the lateral wall of the new vagina. These samples and the amniotic membrane fragments selected for the ultrastructural study were fixed with $3 \%$ glutaraldehyde for at least $2 \mathrm{~h}$. The specimens were postfixed with $1 \% \mathrm{OsO}_{4}$ solution in $0.2 \mathrm{M}$ cacodylate buffer, $\mathrm{pH} \mathrm{7.3,} \mathrm{for} 2 \mathrm{~h}$, rinsed three times in distilled water, cut into $5-\mathrm{mm}^{2}$ fragments, and placed in $2 \%$ uranyl acetate solution. After $24 \mathrm{~h}$ the specimens were rinsed three times with distilled water and serially dehydrated in increasing alcohol concentrations of 70 to $100 \%$ for $10 \mathrm{~min}$ each. The specimens were then treated for $10 \mathrm{~min}$ with a 99\% propylene oxide solution, embedded in 3:1 and 1:1 solutions of propylene oxide and araldite for $60 \mathrm{~min}$ each and further embedded and shaken overnight in pure araldite resin. One-mm thick sections were cut with glass knives using a Reichert-Jung ultramicrotome and stained with $1 \%$ toluidine blue for orientation and light microscopy analysis. The selected areas were then cut into 60$\mathrm{nm}$ sections and stained with lead citrate and uranyl acetate for examination with an EM Philips 300 transmission electron microscope. 


\section{Results}

Patient age ranged from 18 to 28 years (mean, 20.8 years). Preoperative vaginal length ranged from zero (absence) to $3 \mathrm{~cm}$. Most patients were amenorrheic and either incapable of or experiencing difficulty in engaging in sexual activity.

The transmission electron microscopy findings of the reflected amnion epithelium from 10 donors consisted of a sheet of closely apposed cells with numerous microvilli that projected from the apical surface. The cells ranged from cuboidal to low columnar with centrally located nuclei. Variable numbers of cytoplasmic lipid droplets were seen. There were intercellular junctions (desmosomes) and cellular processes at the base of the cells where they attached to the underlying basal lamina. The basal lamina was thick and presented a lattice of undulated collagen fibrils arranged in parallel to the epithelium. The underlying chorion was formed by fibrils mostly arranged in bundles and forming a loose network (Figure 1).

After 8 days, when the latex mold was removed, the amnion could be seen as a distinct layer applied to the vaginal wall with widespread but small areas of congestion. Gross examination revealed no severe inflammatory reaction or necrosis. Sixty days after surgery the vagina was found to be well formed and of satisfactory depth and caliber in all patients.

The transmission electron microscopy findings for the new vaginal epithelium 60 days after amnion grafting consisted of a lining of mature squamous epithelium indicating complete epithelialization (Figure 2). The cells were arranged in layers as in the normal vaginal epithelium, i.e., superficial, intermediate and deep layers. There were intercellular junctions (desmosomes) and cytoplasmic intermediate cytokeratin filaments. The basal lamina was thick and the underlying chorion was formed by loose connective tissue (Figures 2 and 3). In a few

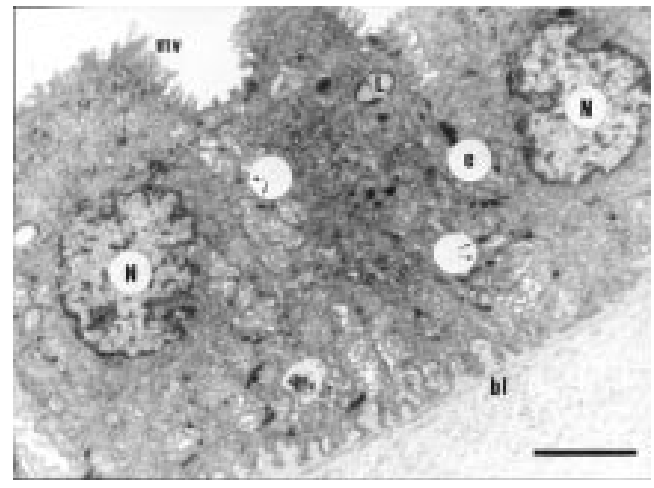

Figure 1 - Transmission electron micrograph of a reflected portion of amnion epithelium (e) showing cuboidal to low columnar cells with centrally located nuclei (N). Variable numbers of cytoplasmic lipid droplets are seen (L) as well as numerous microvilli projecting from the apical surface (mv). bl indicates the basal lamina and arrows indicate desmosomes. Lead citrate and uranyl acetate staining (bar $=1$ $\mu \mathrm{m})$.

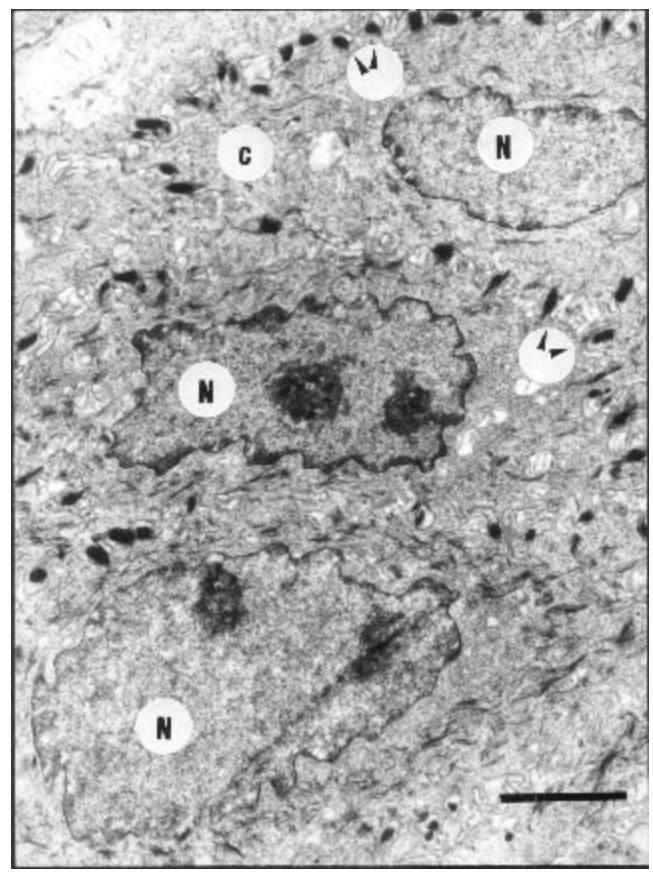

Figure 2 - Transmission electron micrograph of new vaginal epithelium 60 days after application of amnion graft. The cells are characteristic of squamous epithelium with oval nuclei (N), abundant cytoplasm (c) with numerous desmosomes (arrow heads) and tonofilaments. Lead citrate and uranyl acetate staining (bar $=1 \mu \mathrm{m})$.

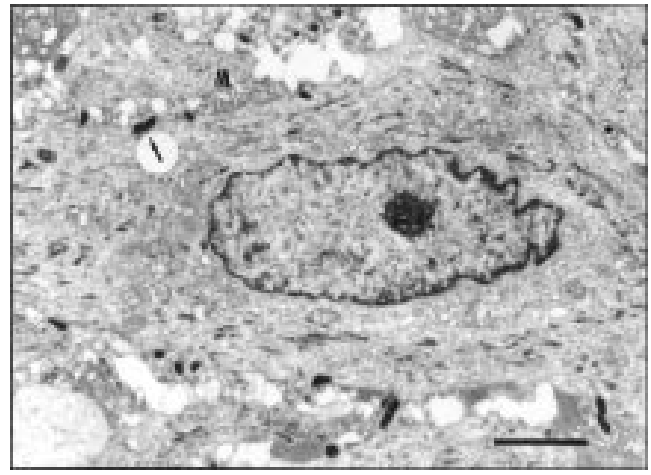

Figure 3 - Transmission electron micrograph of neovagina squamous epithelium with abundant cytoplasm, clearly visible nucleoli and numerous desmosomes (arrow). There are some areas suggesting interdigitation $\left({ }^{*}\right)$ of cells with occasional residual microvilli (M). This evidence suggests a squamous metaplasia of amniotic tissue. Lead citrate and uranyl acetate staining $(\mathrm{bar}=1 \mu \mathrm{m})$. 
cases there was evidence of interdigitation and residual structures compatible with microvilli (Figure 3).

\section{Discussion}

The human amniotic membrane is a readily available cost-free protective biologic dressing for wounds and burns $(2,3)$. It has also been shown to stimulate granulation tissue and new vessel formation in chronic venous leg ulcers by producing angiogenic factors, as demonstrated by Faulk et al. (2). The amnion does not express histocompatibility antigens and there is no evidence of immune rejection when amnion is implanted subcutaneously (9). These features make the amnion a perfect tissue graft for covering the artificially dissected vagina in patients with congenital absence of the vagina.

When an uncontaminated amniotic mesenchymal surface is applied to the raw surface of the dissected vaginal tunnel, it adheres firmly, protects the underlying granulation tissue and facilitates epithelialization. Dhall (4) has reported 5 patients from whom vaginal biopsy specimens were taken at follow-up visits, showing a mature vaginal epi- thelium 8 to 10 weeks after surgery. Ashworth et al. (1) reported 15 other patients with vaginal abnormalities in whom amnion was successfully used.

To date, the present report is the first transmission electron microscopy study of the new epithelium developed from the amniotic membrane applied to a surgically dissected vagina. Sixty days after the application of amnion to the artificially produced vaginal surface, the new vaginal epithelium presented all the normal ultrastructure characteristics of the vagina, including layer arrangement, nucleolus features and cellular organelles.

The new epithelium transformation event and its implications remain obscure. They might be due to metaplasia of the amniotic membrane into squamous epithelium as suggested by some of our findings (Figure 3). However, another possibility is that the human amniotic membrane may play a role in stimulating primitive pluripotent cells in vaginal soft tissue. These cells, under the effect of prostaglandins and interferon that contribute to cellular division and differentiation (10), may be activated to mature towards squamous epithelium.

\section{References}

1. Ashworth MF, Morton KE, Dewhurst Sir John, Lilford RJ \& Bates RG (1986). Vaginoplasty using amnion. Obstetrics and Gynecology, 67: 443-446.

2. Faulk WP, Stevens PJ, Burgos $H$, Matthews R, Bennet JP \& Hsi BL (1980). Human amnion as an adjunct in wound healing. Lancet, 1: 1156-1157.

3. Subrahmanyam M (1995). Amniotic membrane as a cover for microskin grafts. British Journal of Plastic Surgery, 48: 477478.

4. Dhall K (1984). Amnion graft for treatment of congenital absence of the vagina. British Journal of Obstetrics and Gynaecology, 91: 279-282.

5. Morton KE \& Dewhurst CJ (1986). Human amnion in the treatment of vaginal malformation. British Journal of Obstetrics and Gynaecology, 93: 50-54.
6. Moura MD, Ferriani RA, Sá MFS, Wanderley MS, Leite SP \& Soares FA (1994). Epitelização vaginal com membrana amniótica em neovaginoplastia. Revista Brasileira de Ginecologia e Obstetrícia, 16: 135-140.

7. Piazza MJ \& Teixeira AC (1992). Neovaginoplastia pela técnica de Mclndoe com o emprego de membrana amniótica: estudo de 15 pacientes. Revista Brasileira de Ginecologia e Obstetrícia, 14: 224-226.

8. Mclndoe AH \& Bannister JB (1938). An operation for the cure of congenital absence of the vagina. Journal of Obstetrics and Gynaecology of the British Empire, 45: 490-494.
9. Akle CA, Welsh Kl, Adinolfi M, Leibowitz M \& McColl I (1981). Immunogenicity of human amniotic epithelial cells after transplantations into volunteers. Lancet, 2: 1003-1005.

10. Neumark T, Premecz G, Markovits A Neumoller J, Ferencz G, Bagi G, Fnides I \& Eberl R (1990). Ultrastructural changes of human amniotic cells induced by human interferon-alpha. International Journal of Tissue Reactions, 12: 291-297. 